\title{
Anaphylaxis and neuromuscular blocking agents
}

\author{
Ana Vaz, Maria José Arede, Eduarda Figueiredo, José Pedro Assunção
}

Centro Hospitalar Tondela-Viseu, CHTV, Portugal

Euroanaesthasid 18

\section{Background}

Anaphylaxis is a severe life-threatening allergic reaction that can occur during anesthesia. It has an incidence of 0,05-2\%, in which $60-70 \%$ of cases are secondary to neuromuscular blocking agents with either rocuronium or succinylcholine being most commonly implicated(1). An early recognition and a fast management are fundamental, as the investigation with immunoallergology tests for all neuromuscular blocking agents.

\section{Case Report}

A 64 year-old woman, ASA II, BMI normal, with a history of perioperative anaphylactic reaction, in 2007, in France, after administration of propofol, fentanyl, suxamethonium and cisatracurium (information brought in card). Patient scheduled for an urgent laparotomy due to an intestinal occlusion.

Before anesthetic induction, was given hydrocortisone. Shortly after administration of fentanyl, thiopental and rocuronium, and intubation, patient became profoundly hypotensive, tachycardic and developed a diffuse rash. Further, fluidotherapy, sympathomimetic, hydrocortisone and clemastine were given. At the end of the surgery, three hours later, patient was hemodynamically stable and regression of the rash was observed. After decurarization confirmed, patient was awakened, extubated and transferred to the PACU, where remained for two hours without complications. Subsequently, immunoallergology collaboration was requested. One week after that surgery, she underwent on a urgent laparotomy for lysis of abdominal adhesions, under general anesthesia with fentanyl, propofol and ketamine, this time without incidents.

The immunoallergology tests (skin tests with muscle relaxants present in our hospital) were performed 7 weeks after the reaction (53 days). The results were positive for suxamethonium, rocuronium and cisatracurium and negative to vecuronium.

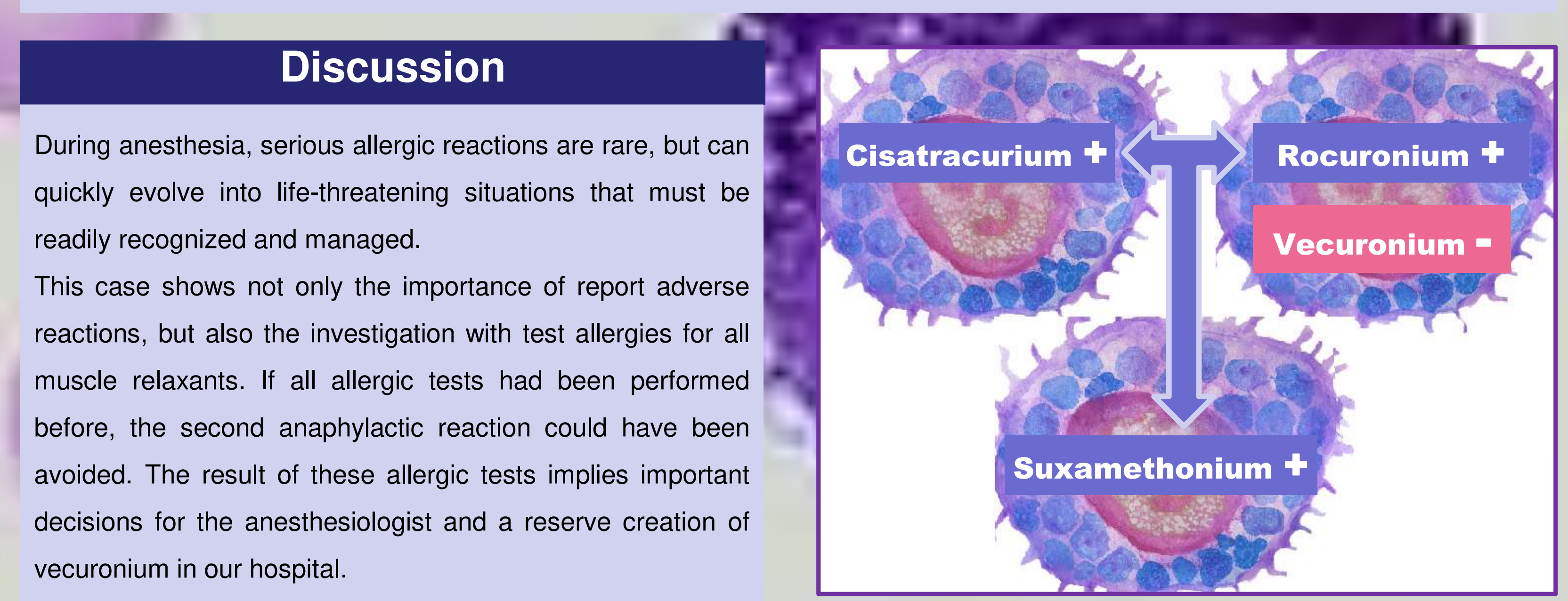

\section{Learning Points}

In a patient with a history of neuromuscular relaxants allergy, it is important to perform tests on all the others.

References: 1- Peroni DG1,et all,Muscle relaxants allergy,Int J Immunopathol Pharmacol.2011 Jul-Sep;24(3 Suppl):S35-46 (PubMed). 